\title{
Role of Interleukin 17A in Aortic Valve Inflammation in Apolipoprotein E-deficient Mice*
}

\author{
Fa-yuan LIU ${ }^{\dagger}$, Peng BAI ${ }^{\dagger}$, Ye-fan JIANG ${ }^{\dagger}$, Nian-guo DONG ${ }^{1}$, Geng $\mathrm{LI}^{1}$, Chong CHU ${ }^{1 \#}$ \\ Department of Cardiovascular Surgery, Union Hospital, Tongji Medical College, Huazhong University of Science and \\ Technology, Wuhan 430022, China
}

(C) The Author(s) 2020

\begin{abstract}
Summary: Interleukin 17A (IL17A) is reported to be involved in many inflammatory processes, but its role in aortic valve diseases remains unknown. We examined the role of IL17A based on an $\mathrm{ApoE}^{-/-}$mouse model with strategies as fed with high-fat diet or treated with IL17A monoclonal antibody (mAb). 12 weeks of high-fat diet feeding can elevate cytokines secretion, inflammatory cells infiltration and myofibroblastic transition of valvular interstitial cells (VICs) in aortic valve. Moreover, diet-induction accelerated interleukin 17 receptor A (IL17RA) activation in VICs. In an IL17A inhibition model, the treatment group was intra-peritoneally injected with anti-IL17A $\mathrm{mAb}$ while controls received irrelevant antibody. Functional blockade of IL17A markedly reduced cellular infiltration and transition in aortic valve. To investigate potential mechanisms, NF- $\kappa$ B was co-stained in IL17RA ${ }^{+}$VICs and IL17RA ${ }^{+}$macrophages, and further confirmed by Western blotting in VICs. High-fat diet could activate NF- $\kappa$ B nuclear translocation in IL17RA ${ }^{+}$VICs and IL17RA macrophages and this process was depressed after IL17A mAb-treatment. In conclusion, highfat diet can lead to IL17A upregulation, VICs myofibroblastic transition and inflammatory cells infiltration in the aortic value of $\mathrm{ApoE}^{-/-}$mice. Blocking IL17A with IL17A mAb can alleviate aortic valve inflammatory states.
\end{abstract}

Key words: aortic valve inflammation; interleukin 17A; NF- $\kappa \mathrm{B}$ pathway; intensity correlation analysis; colocalization

Aortic valve stenosis remains as the most prevalent valvular disease in Western countries ${ }^{[1]}$, affecting over $25 \%$ of all patients over the age of $65^{[2]}$. Currently, there is no effective pharmacological therapy for aortic valve stenosis and surgical or interventional valve replacement serves as the only curative treatments. However, in patients who underwent transcatheter aortic valve replacement, up to $30 \%$ had oxygen dependence $^{[3]}$ and $60 \%$ had significant lung disease, which has been associated with increased morbidity and mortality ${ }^{[4]}$. Others reported unexpected side effects including chronic kidney disease and liver disease, all of which are considered to contribute to deaths after

Fa-yuan LIU, E-mail: liufayuan@hust.edu.cn; Peng BAI, E-mail: baipenghust@gmail.com; Ye-fan JIANG, E-mail: 422193780@qq.com

†These authors contributed equally to this work.

"Corresponding author, E-mail: chongchu@hust.edu.cn

${ }^{*}$ This project was supported by grants from the National Key Research and Development Program of China (No. 2016YFA0101100), National Natural Science Foundation of China (No. 81700339 and No. 31330029), and Scientific Research Training Program for Young Talents sponsored by Union Hospital, Tongji Medical College, Huazhong University of Science and Technology. aortic valve surgery ${ }^{[5]}$.

Inflammation plays a key role in aortic valve stenosis, ultimately leading to aortic valve calcification. Low density lipoprotein (LDL) cholesterol has also been suggested to underlie the progression of inflammatory aortic valve stenosis ${ }^{[6]}$. High-fat diet (HFD) can induce significant aortic valve calcification in $\mathrm{ApoE}^{-/-}$mice $^{[7]}$, significantly increasing transvalvular peak jet velocity and markedly decreasing aortic valve area (AVA) and AVA index. Similarly, significant calcium deposits in aortic valve of HFD-treated $\mathrm{ApoE}^{-/-}$ mice by alizarin red staining ${ }^{[7]}$. Currently, ApoE ${ }^{-/-}$mice fed with HFD are the common animal models for aortic valve inflammation study ${ }^{[7-9]}$.

In recent years, interleukin 17A (IL17A) was reported to coordinate local tissue inflammation via inducing the release of proinflammatory cytokines and neutrophil-mobilizing chemokines in several cell families $^{[10,11]}$. And recent studies implicate that IL17A can also accelerate atherosclerotic plaque development via its receptor IL17RA ${ }^{[12,13]}$, and vascular inflammation decreased in the IL17A-deficient mice ${ }^{[14]}$. Besides, IL17A is primarily produced by Th17 cells, a subset of $\mathrm{CD}^{+} \mathrm{T}$ cells, and Th17 cells have also been observed in atherosclerotic plaques both in human and animals ${ }^{[15]}$. 
These findings have stroked our view for uncovering the character of IL17A/IL17RA performance in aortic valve inflammation.

We therefore sought to accurately examine the role of IL17A in aortic valve inflammation using HFD mouse models, and blocking IL17A with an anti-IL17A antibody $(\mathrm{mAb})$ in $\mathrm{ApoE}^{-/}$mice.

\section{MATERIALS AND METHODS}

\subsection{Affymetrix Microarray Data and Data Analysis}

Microarray data of GSE77287 were obtained from the Gene Expression Omnibus (GEO; http://www. ncbi.nlm.nih.gov/geo/) database. Calcific aortic valve tissues were extracted from patients with calcific aortic valve disease (CAVD) who underwent aortic valve replacement, and normal controls were obtained from non-CAVD cardiac transplant recipient hearts ${ }^{[16]}$. Gene expression profiles of three samples of calcific aortic valves and three age-matched normal controls were performed using Affymetrix Gene Chip microarrays ${ }^{[16]}$. The raw data and annotation files were downloaded for subsequent analysis, based on Affymetrix Human Gene 2.0 ST Array. The original data were preprocessed with background correction, normalization and calculating expression using the 'affy' package in $\mathrm{R}$ (version 3.4.2). Heatmap and PPI network were applied as indicated ${ }^{[17]}$.

\subsection{Animals}

Eight-week (wk)-aged male $\mathrm{ApoE}^{-/}$mice were purchased from the local experimental animal center (Wuhan, China). Mice were housed in a controlled environment $\left(20 \pm 2^{\circ} \mathrm{C}, 12 \mathrm{~h} / 12 \mathrm{~h} \mathrm{light} /\right.$ dark cycle $)$ and had free access to water and diet. The experiments were randomly assigned into four groups: (1) normal group, 8-wk-aged $\mathrm{ApoE}^{-/-}$mice receiving normal diet (ND) for 12 weeks; (2) HFD group, 8-wk-aged ApoE-/mice receiving $0.25 \%$ HFD for 12 weeks; (3) IL17A mAb-treated group, 8-wk-aged $\mathrm{ApoE}^{-/-}$mice receiving anti-mouse IL17A mAb (100 $\mu \mathrm{g}$; R\&D Systems, USA) intraperitoneally (i.p.) once a week and $0.25 \%$ HFD for 12 weeks; (4) irrelevant IgG-treated group (control group), 8-wk-aged ApoE ${ }^{-/-}$mice receiving irrelevant $\operatorname{IgG}(100 \mu \mathrm{g}$; R\&D Systems, USA) i.p. once a week and $0.25 \%$ HFD for 12 weeks.

All the mice were sacrificed at 20th week, and aortic valves were collected and stored at $-80^{\circ} \mathrm{C}$ until cutting. All the animal procedures were performed according to the Helsinki Declaration and institutional guidelines at the laboratory animal center of Huazhong University of Science and Technology.

\subsection{Immunohistochemistry}

The extent of aortic valve was assessed by immunohistochemistry. Lipid depositions were assessed following Oil Red O staining and nuclei were stained with hematoxylin. $\mathrm{CD}^{+} \mathrm{T}$ cells, valvular interstitial cells (VICs) and macrophages were stained in aortic valves. Briefly, after blocking by horse serum (Sigma Aldrich, USA), samples were reacted with rabbit antimouse CD4 (1:500 dilution, Abcam, ab183685, UK), FITC-conjugated mouse anti-mouse smooth muscle cell $\alpha$-actin ( $\alpha$ SMA, 1:500, Sigma Aldrich, F3777, USA), rat anti-mouse CD68 (1:100 dilution, Serotec, MCA1957, UK), rat anti-mouse IL17A (1:100 dilution, R\&D, MAB721, USA), goat anti-mouse IL17RA(1:100 dilution, R\&D, AF448, USA) and rabbit anti-mouse NF-kB (p65) (1:100 dilution, Santa Cruz, sc-372, USA) antibodies at $4{ }^{\circ} \mathrm{C}$ overnight in the dark, respectively. After incubation with secondary antibody for $1 \mathrm{~h}$ at room temperature in the dark, nuclei were counterstained with DAPI (Vector Laboratories, USA). Images were recorded and analyzed with Image J software.

\subsection{Quantitative Assessment of Colocalization}

Intensity correlation analysis (ICA ${ }^{[18]}$ was performed on immunofluorescent images to assess the percentage of labelled $\mathrm{IL}_{17 \mathrm{~A}^{+}} \mathrm{CD}^{+} \mathrm{T}$ cells (Th17 cells), IL17RA ${ }^{+} \mathrm{CD}^{+}{ }^{+} \mathrm{T}$ cells, IL17RA ${ }^{+}$VICs and IL17RA ${ }^{+}$macrophages in aortic valves. Briefly, WCIF Image $\mathrm{J}$ software was used to determine colocalized red and green pixel areas. We obtained intensity correlation quotient (ICQ), $\sum \mathrm{N}\left(\mathrm{A}_{\mathrm{i}}-\mathrm{a}\right)\left(\mathrm{B}_{\mathrm{i}}-\mathrm{b}\right)$ value, with dependent staining $0<\mathrm{ICQ} \leq+0.5$. The percentage of colocalization was calculated relative to total aortic valve area for that field. Online manual for correlation analysis is referred to $h t t p: / / w w w f a c i l i t i e s$. uhnresearch.ca/wcif/imagej/colour_analysis.htm.

\subsection{Plasma Lipid and ELISA Analysis}

Total plasma cholesterol and triglycerides were enzymatically measured using the Cholesterol/ Triglyceride Assay Kit (Roche-Hitachi, Switzerland) according to the manufacturer's instructions. Serum IL17A and other cytokines levels were measured with IL17A, IL6 and TNF- $\alpha$ ELISA (Elabscience, China) according to the manufacturer's protocols.

\subsection{Cell Harvest and Western Blotting Analysis}

VICs were isolated from murine aortic valve leaflets and cultured as previously described with some modifications ${ }^{[16]}$. Briefly, aortic valve leaflets were digested in collagenase $(2.5 \mathrm{mg} / \mathrm{mL}$ in M199 medium) for $30 \mathrm{~min}$ at $37^{\circ} \mathrm{C}$. After removing immune and endothelial cells by vortex, a milder solution of collagenase medium was used $(0.8 \mathrm{mg} / \mathrm{mL})$ for $3 \mathrm{~h}$ at $37^{\circ} \mathrm{C}$. Cells were collected by centrifugation at $1200 \times \mathrm{g}$ for $5 \mathrm{~min}$, and then resuspended and cultured in DMEM containing $4.5 \mathrm{~g} / \mathrm{L}$ glucose, penicillin $\mathrm{G}$, streptomycin, and $10 \%$ fetal bovine serum. Cells were harvested and homogenized in RIPA Lysis and Extraction Buffer with protease and phosphatase inhibitor cocktails (Pierce, USA). Nuclear proteins were prepared using NE-PER Nuclear Extraction Reagents (Pierce, USA). All these procedures were performed according to the manufacturer's protocols. Equal amounts of protein 
were resolved by SDS-PAGE (10\% resolving gel with $4 \%$ stacking) and transferred to PVDF membranes. Membranes were blocked with buffer containing 10\% non-fat milk and $5 \%$ BSA in TBS-T $(50 \mathrm{mmol} / \mathrm{L}$ Tris$\mathrm{HCl}, \mathrm{pH} 8.0,150 \mathrm{mmol} / \mathrm{L} \mathrm{NaCl}$, and $0.1 \%$ Tween-20), and then incubated with NF-kB (p65) (1:500 dilution, Bioworld Technology, T429, USA) and $\beta$-actin (1:1000 dilution, Santa Cruz, sc-8432, USA). Blots were washed in TBS-T and incubated with appropriate HRP-conjugated secondary antibodies. The immune complexes were then visualized using ECL reagent (Beyotime, China). Image J software was used to analyze the area and density of protein band.

\subsection{Quantitative Real-time RT-PCR Validation}

The target genes IL17A and IL17RA were validated by quantitative real-time RT-PCR. Total RNA was isolated and cDNA was transcribed using PrimeScript RT Reagent Kit with gDNA Eraser (TaKaRa, Japan) according to the manufacturer's instructions. Primers were searched from Primerbank (https://pga.mgh. harvard.edu/primerbank/) and listed in table 1. All data were normalized to GAPDH. $2^{-\triangle \Delta C t}$ methods were recruited to calculate the relative expression level.

\subsection{Statistics}

Statistical calculations were performed using GraphPad Prism 7 (GraphPad Software Inc., USA). With unpaired Student's $t$-test or sign test of the normal approximation means, data were expressed as mean \pm SEM. The $P$ value $<0.05$ was considered significant.

\section{RESULTS}

\subsection{Up-regulation of IL17A-IL17RA Axis in Aortic Valve of HFD Apo $\mathrm{E}^{-/-}$Mice}

Firstly, IL17A-IL17RA-related genes from the analysis of GSE77287 were selected based on PPI network. Relative expression of these genes was shown in fig. $1 \mathrm{~A}-1 \mathrm{C}$.

To determine whether IL17A and IL17RA levels are altered in aortic valve inflammation, $\mathrm{ApoE}^{-/-}$mice models were established with HFD and aortic valves were collected from both ND and HFD ApoE ${ }^{-/}$mice. Oil Red $\mathrm{O}$ staining verified the histomorphology changes. Prominent lipid-rich areas were observed and mostly located in the superficial fibrosa layer (fig. 1D). Furthermore, we analyzed the composition of aortic valves. Increased VICs myofibroblastic differentiation was companied by enhanced $\mathrm{CD}^{+} \mathrm{T}$ cells and macrophages infiltration in aortic valve (fig. $1 \mathrm{G}$ and $1 \mathrm{I}$, for VICs, ND: $8.18 \% \pm 2.70 \%$, HFD: $17.22 \% \pm 4.56 \%$, $P<0.01$; fig. $1 \mathrm{~F}$ and $1 \mathrm{I}$, for $\mathrm{CD}^{+} \mathrm{T}$ cells, ND: $2.77 \% \pm 1.06 \%$, HFD: $9.06 \% \pm 2.60 \%, P<0.01$; fig. $1 \mathrm{H}$ and $1 \mathrm{I}$, for macrophages, ND: $3.38 \% \pm 1.19 \%$, HFD: $7.65 \% \pm 2.52 \%, P<0.05)$, indicating the valves suffered from inflammation. Previous literatures also supported activation of myofibroblastic VICs, $\mathrm{CD}^{+} \mathrm{T}$ cells and macrophages in inflammatory aortic valve ${ }^{[19,20]}$.

Furtherly, as shown in fig. 1E and table 2, results suggested an increase of IL17A ${ }^{+} \mathrm{CD} 4^{+} \mathrm{T}$ cells (Th17 cells) in HFD-induced aortic valve (fig. 1E), as well as serum cytokines concentration, including IL17A, IL6 and TNF- $\alpha$ (table 2). A visual inspection of the staining in aortic valve provided suggestive evidence for colocalization of IL17A with CD4. Moreover, the ICA analysis was consistently in favor of such an association, concerning both the staining amplitude $(\mathrm{A}-\mathrm{a})(\mathrm{B}-\mathrm{b})$ plots and positive ICQ values $(\mathrm{ICQ}=+0.15$, $P_{\text {sign test }}<0.001$, fig. 1E).

Double immunofluorescent staining indicated IL17RA colocalized with CD4 (fig. 1F), aSMA (fig. $1 \mathrm{G}$ ) and CD68 (fig. 1H) in aortic valve. ICA analysis

Table 1 The primers of IL17A-IL17RA-related genes

\begin{tabular}{lll}
\hline Gene & Forward & Reverse \\
\hline IL17RA & 5'-TGACTGTTTGAGACACGCT-3' & 5'-ATGTAGTCTGCAACTGGCT-3' \\
IL17A & 5'-AGACCTCATTGGTGTCACTG-3' & 5'-CTGGATTTCGTGGGATTGTG-3' \\
TGFB1 & 5'-GAGCCCGAAGCGGACTACTA-3' & 5'-TGGTTTTCTCATAGATGGCGTTG-3' \\
IL6 & 5'-CTGCAAGAGACTTCCATCCAG-3' & 5'-AGTGGTATAGACAGGTCTGTTGG-3' \\
IL10 & 5'-TTAATAAGCTCCAAGACCAAGG-3' & 5'-CATCATGTATGCTTCTATGCAG-3' \\
STAT1 & 5'-GATTTAATCAGGCTCAGTCGG-3' & 5'-TTCTGACTTTACTGTCAAGCTC-3' \\
IL17C & 5'-TCTGCTGAGGAATTATCTCACGG-3' & 5'-GTTCCAGCTAGAGGTCCTTCA-3' \\
STAT3 & 5'-CGCACTTTAGATTCATTGATGC-3' & 5'-AGGTGAGGGACTCAAACTG-3' \\
NFKBIA & 5'-CAACTACAATGGCCACACG-3' & 5'-GAGCATTGACATCAGCACC-3' \\
IL17RC & 5'-AAAGTTTGGAGGAGCAGCT-3' & 5'-ATATACCACAGAGCCCACAG-3' \\
IL25 & 5'-ACAGGGACTTGAATCGGGTC-3' & 5'-TGGTAAAGTGGGACGGAGTTG-3' \\
IL17B & 5'-GAGTAAAGCCCTACGCTCGAA-3' & 5'-CTCCTCTTGTTGGACAACCAC-3' \\
TRAF3IP2 & 5'-AGCATTCCCGTGGAGGTTG-3' & 5'-GGGCAGGTGGTTCCAATTC-3' \\
IL17RE & 5'-AGCTCTGCTTCAAGTTCTC-3' & 5'-TTACATTCCAGGATGTGAGAG-3' \\
IL23A & 5'-GAAGAGACTACAAATGATGTTCCC-3' & 5'-CAAGCAGAACTGACTGTTGTC-3' \\
IL17RB & 5'-TGGGAAACAGATACATGGCT-3' & 5'-GTTTCTTCTGGTGTGGCTC-3' \\
IL17F & 5'-AATTACACTGTCACTTGGGAC-3' & 5'-TCCTTGAGCATTGATGCAG-3' \\
IL2 & 5'-GAGGAAGTGCTAAATTTAGCTC-3' & 5'-TTCAGATCCCTTTAGTTCCAG-3' \\
GAPDH & 5'-AGGTCGGTGTGAACGGATTTG-3' & 5'-TGTAGACCATGTAGTTGAGGTCA-3' \\
\hline
\end{tabular}




\begin{tabular}{lccc}
\multicolumn{4}{c}{ Table 2 Lipid profile and serum levels of cytokines } \\
\hline Parameters & ND ApoE $^{-1}(n=7)$ & HFD ApoE $^{-/}(n=7)$ & $P$ value \\
\hline Triglycerides $(\mathrm{mg} / \mathrm{dL})$ & $92.64 \pm 31.16$ & $143.23 \pm 68.32$ & n.s. \\
Total cholesterol $(\mathrm{mg} / \mathrm{dL})$ & $387.97 \pm 196.60$ & $512.73 \pm 213.62$ & 0.05 \\
IL17A $(\mathrm{pg} / \mathrm{mL})$ & $92.45 \pm 36.13$ & $153.59 \pm 33.50$ & $<0.05$ \\
IL6 $(\mathrm{pg} / \mathrm{mL})$ & $474.82 \pm 5.70$ & $623.21 \pm 4.77$ & $<0.01$ \\
TNF- $\alpha(\mathrm{pg} / \mathrm{mL})$ & $445.04 \pm 39.12$ & $515.82 \pm 13.73$ & $<0.01$ \\
\hline
\end{tabular}

On the day of tissue harvesting ( 20 weeks), serum levels of cytokines $(\mathrm{pg} / \mathrm{mL})$ as well as lipid profile in the serum were determined by ELISA. Data are expressed as mean \pm standard error of the mean. n.s., not significant
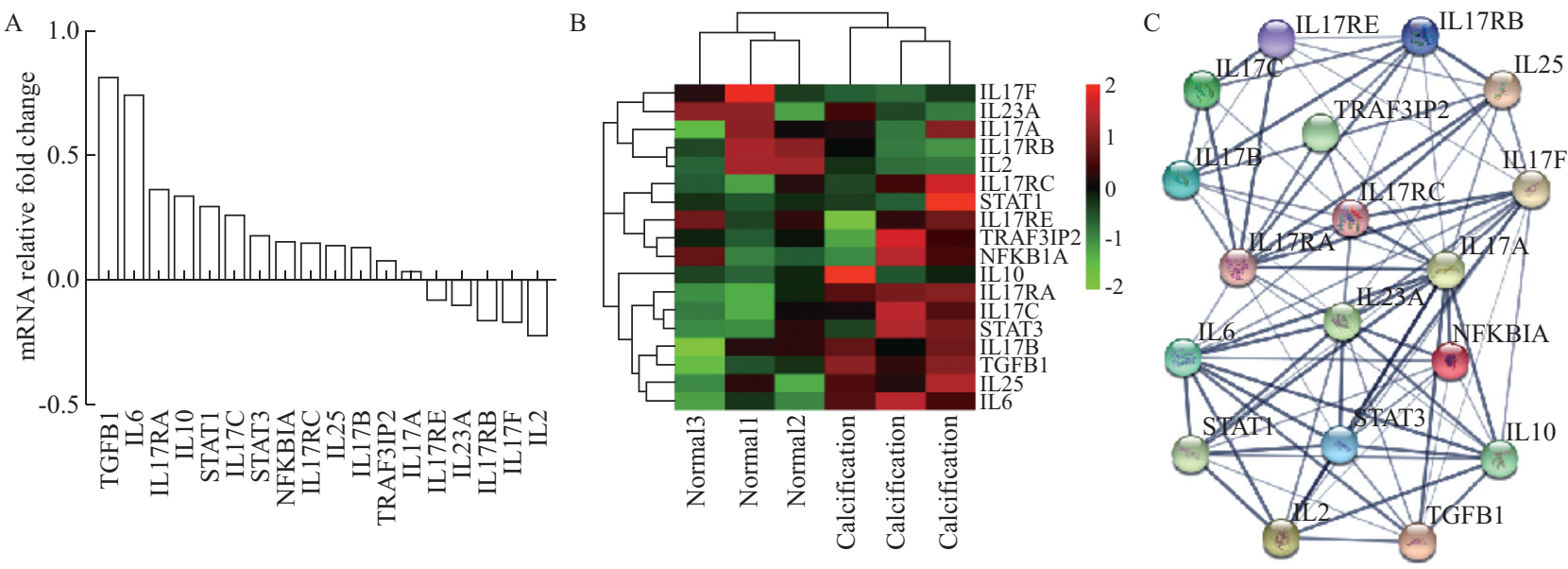

$\mathrm{D}$

Normal diet $\mathrm{ApoE}^{-/-} 20 \times$
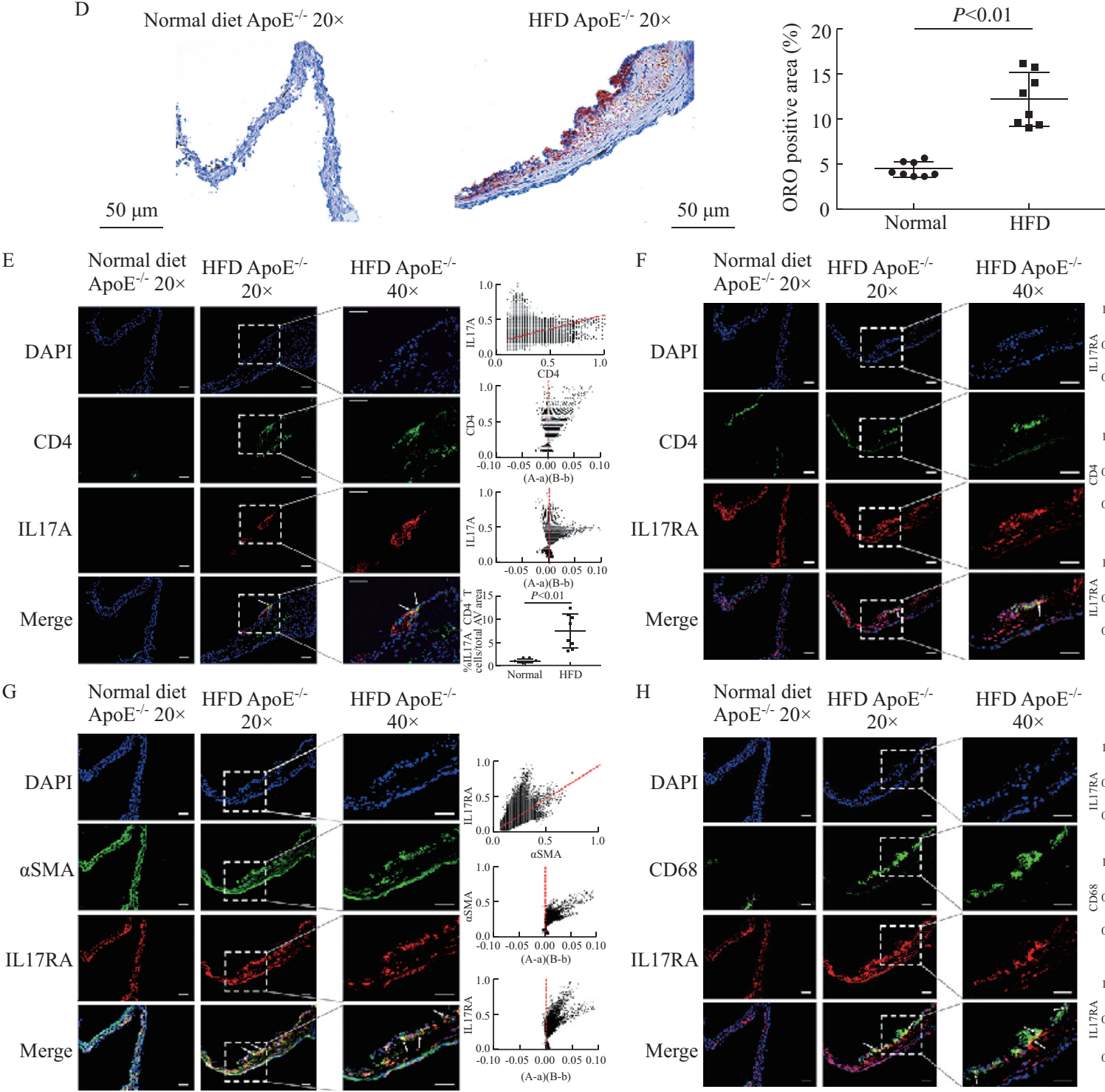

$\mathrm{H}$

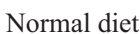
$\mathrm{ApoE}^{-/ /} 20 \times$
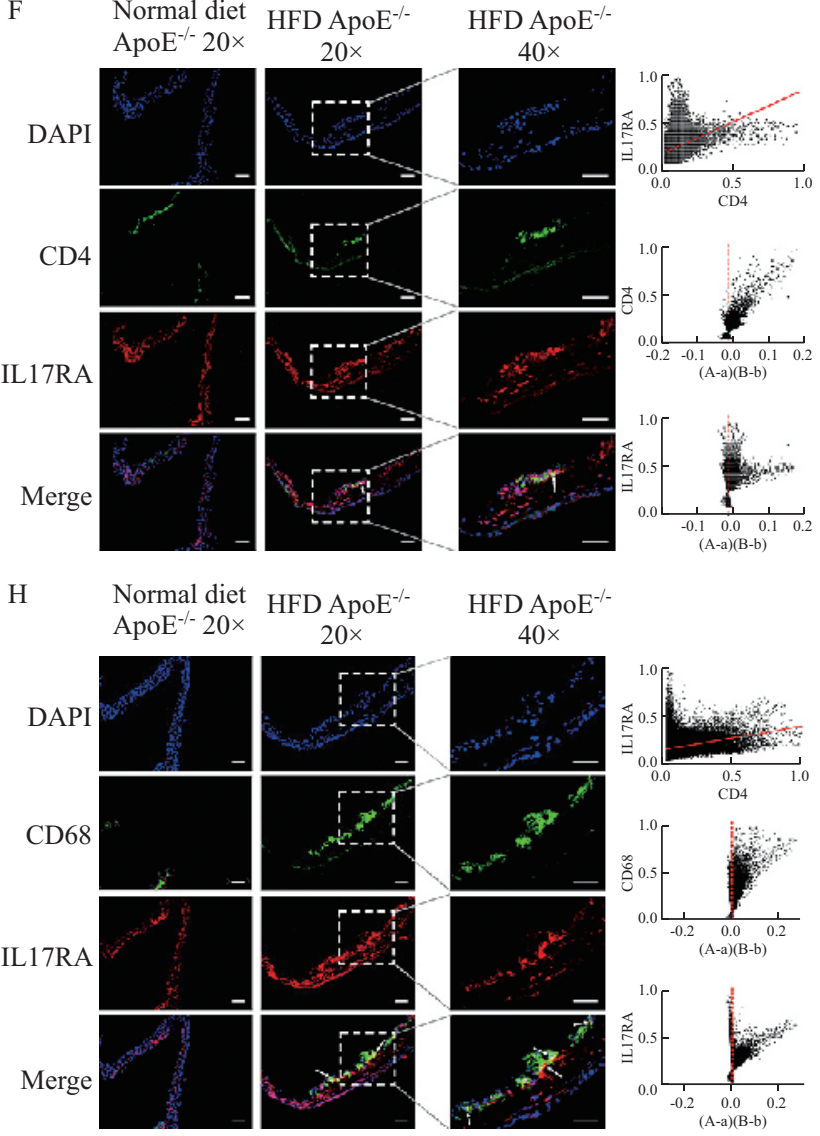

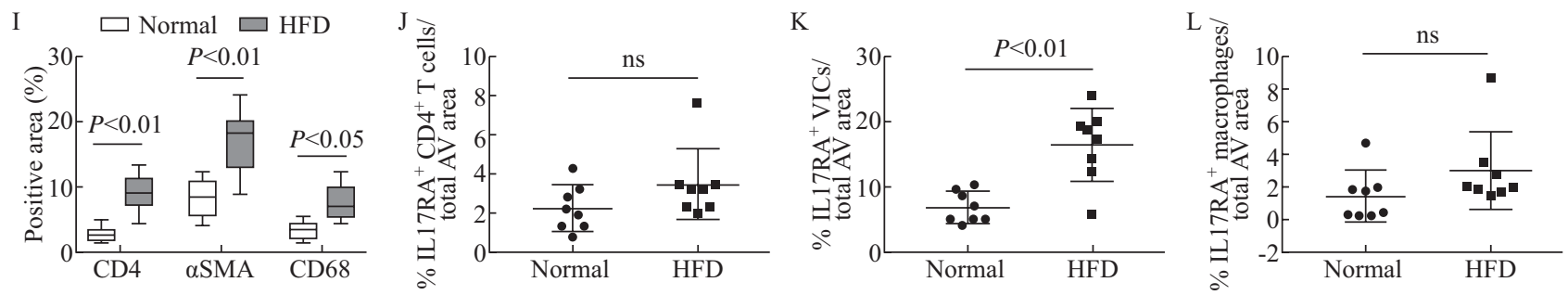

Fig. 1 Up-regulation of IL17A-IL17RA axis in aortic valve of high-fat diet ApoE ${ }^{-/}$mice

Gene expression of aortic valve from patients with calcific aortic valve disease and age-matched normal controls was analyzed by Affymetrix array. A: relative expression of IL17A-IL17RA-related genes. The expression levels of TGFB1, IL6 and IL17RA mRNA were significantly increased. B and C: Heatmap (B) and PPI network (C) of IL17A-IL17RA-related genes. ApoE ${ }^{-/-}$mice $^{-}$ were fed with a normal diet (ND) or high fat diet (HFD) for 12 weeks. D: Oil Red O staining verified the prominent lipid-rich areas observed in aortic valve superficial fibrosa layer $(n=8$ per group; $P<0.01)$. E: Immunofluorescence staining of IL17 $\mathrm{A}^{+}(\mathrm{red})$ $\mathrm{CD}^{+}$(green) T cells in aortic valve of ND and HFD mice was analyzed by colocalization analysis $(n=8$ per group; $P<0.01)$. The aortic valves had also been isolated for Intensity Correlation Analysis for IL17A compared with CD4. Top panel. scatter plot of pixel staining intensities for CD4 and IL17A. Center panels: ICA plots of CD4 and IL17A staining intensities against their corresponding (A-a) and (B-b) values. ICQ $=+0.15, P_{\text {sign test }}<0.001 . \mathrm{F}-\mathrm{H}$ : double immunofluorescence staining images of IL17RA/ CD4 (F), IL17RA/ $\alpha$ SMA $(G)$, and IL17RA/CD68 (H). I-L: The quantification data were displayed respectively ( $n=8$ per group). Quantification of $\mathrm{CD}^{+} \mathrm{T}$ cells $\left(\mathrm{CD} 4^{+}\right.$area), macrophages $\left(\mathrm{CD} 8^{+}\right.$area) and VICs $\left(\alpha \mathrm{SMA}^{+}\right.$area) shows that HFD significantly increased infiltration of $\mathrm{CD}^{+} \mathrm{T}$ cells $(P<0.01)$ and macrophages $(P<0.05)$ as well as transition of VICs myofibroblast $(P<0.01)$ than ND in ApoE ${ }^{-/}$mice. Colocalization area results suggested that IL17RA ${ }^{+}$VICs $(\mathrm{K})$ accumulated in aortic valve of HFD mice $(P<0.01)$, while IL17RA ${ }^{+} \mathrm{CD} 4^{+} \mathrm{T}$ cells $(\mathrm{J})$ and IL17RA ${ }^{+}$macrophages $(\mathrm{L})$ showed no significant difference from ND mice. $20 \times$ magnification, scale bar $=40 \mu \mathrm{m} ; 40 \times$ magnification, scale bar $=50 \mu \mathrm{m}$

demonstrated a strongly dependent staining patterns with IL17RA/CD4, IL17RA/ $\alpha$ SMA and IL17RA/ $\mathrm{CD} 68$ in aortic valve $\left(\mathrm{ICQ}_{\mathrm{L} 17 \mathrm{R} / \mathrm{CD} 4}=+0.25 ; \mathrm{ICQ}_{\mathrm{IL17RA} /}\right.$ ${ }_{\alpha . S M A}=+0.27 ; \mathrm{ICQ}_{\mathrm{IL} 17 \mathrm{RA} / \mathrm{CD} 68}=+0.19 ;$ all $\left.P_{\text {sign test }}<0.001\right)$. Meanwhile, in comparison with ND aortic valve, IL17RA ${ }^{+}$VICs accumulated in HFD aortic valve (fig. $1 \mathrm{~K})$. However, IL17RA ${ }^{+} \mathrm{CD}^{+} \mathrm{T}$ cells and macrophages showed no significant difference (fig. 1J and 1L).

\subsection{Blocking IL17A Alleviated Inflammatory State of Aortic Valve of HFD ApoE ${ }^{-/-}$Mice}

Twelve-week HFD increased IL17A expression in aortic valve. As this potential point, we next sought to address the contribution of IL17A to valvular cells by inactivating IL17A with IL17A mAb. IL17A mAbtreated mice were introduced as a validated mouse model ${ }^{[14,21]}$. Treatment with IL17A mAb resulted in significantly reduced serum cytokines secretion (e.g., IL17A, IL6, TNF- $\alpha$ ) (table 3). Staining with Oil Red $\mathrm{O}$ in IL17A mAb-treated mice presented lower amounts of lipid-rich areas (fig. 2A). Furthermore, $\alpha \mathrm{SMA}^{+}$area in IL17A mAb-treated and control groups was $12.19 \% \pm 2.37 \%$ and $20.65 \% \pm 3.99 \%$ respectively $(P<0.01$, fig. $2 \mathrm{D}$ and $2 \mathrm{G})$. Meanwhile, decreases in
$\mathrm{CD} 8^{+}$and $\mathrm{CD} 4^{+}$area were detected in the IL17A mAbtreated group compared to the control group $\left(\mathrm{CD}^{+}\right.$: $4.95 \% \pm 2.36 \%$ vs. $9.04 \% \pm 3.50 \%, P<0.05$, fig. $2 \mathrm{C}$ and 2G; CD68 : $2.79 \% \pm 1.85 \%$ vs. $7.21 \% \pm 3.35 \%, P<0.05$, fig. $2 \mathrm{E}$ and $2 \mathrm{G})$. The double immunofluorescent staining revealed a reduced number of $\mathrm{IL}_{17 \mathrm{~A}^{+}} \mathrm{CD} 4^{+}$ T cells $(P<0.05)$ (fig. $2 \mathrm{~B}$ and $2 \mathrm{~F})$ and IL17RA ${ }^{+}$VICs $(P<0.01)$ (fig. $2 \mathrm{D}$ and $2 \mathrm{H})$. However, IL17RA ${ }^{+} \mathrm{CD} 4^{+}$ $\mathrm{T}$ cells (fig. 2I) and IL17RA ${ }^{+}$macrophages (fig. 2J) in IL17A mAb-treated groups had no significant difference from control group. Taken together, these data demonstrated that blocking IL17A could lead to a reduction of inflammatory cells in aortic valve as well as drop of IL17RA ${ }^{+}$VICs.

\subsection{IL17A/IL17RA Were Involved in Aortic Valve Inflammation through NF-кB Signaling Pathway}

Aortic valve inflammation is always associated with NF- $\kappa \mathrm{B}$ nuclear translocation ${ }^{[22]}$. We focused on identifying IL17A in NF- $\kappa B$ signaling pathway of aortic valve inflammation. To confirm our conjecture, NF- $\kappa B$ was stained with $\alpha$ SMA-IL17RA-DAPI (fig. $3 \mathrm{~A}$ and 3C) and CD68-IL17RA-DAPI (fig. 3B and $3 \mathrm{D})$. By counting NF- $\mathrm{\kappa B}$ fluorescent positive area or

Table 3 Lipid profile and serum levels of cytokines

\begin{tabular}{lccc}
\hline Parameters & IL17A mAb-treated $(n=8)$ & Control $(n=8)$ & $P$ value \\
\hline Triglycerides $(\mathrm{mg} / \mathrm{dL})$ & $147.64 \pm 76.16$ & $150.30 \pm 50.94$ & n.s. \\
Total cholesterol $(\mathrm{mg} / \mathrm{dL})$ & $395.39 \pm 102.23$ & $439.02 \pm 130.62$ & n.s. \\
IL17A $(\mathrm{pg} / \mathrm{mL})$ & $48.18 \pm 26.82$ & $151.20 \pm 52.80$ & $<0.01$ \\
IL6 $(\mathrm{pg} / \mathrm{mL})$ & $474.15 \pm 12.19$ & $571.01 \pm 44.16$ & $<0.01$ \\
TNF- $\alpha(\mathrm{pg} / \mathrm{mL})$ & $475.94 \pm 9.22$ & $569.88 \pm 20.61$ & $<0.01$ \\
\hline
\end{tabular}

On the day of tissue harvesting (20 weeks), serum levels of cytokines $(\mathrm{pg} / \mathrm{mL})$ as well as lipid profile in the serum were determined by ELISA. Data are expressed as mean \pm SEM. n.s., not significant 
A

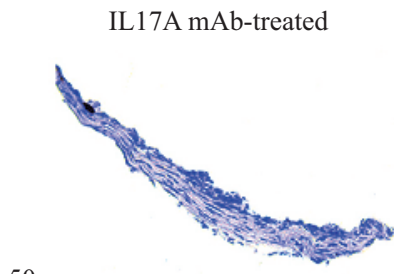

$50 \mu \mathrm{m}$
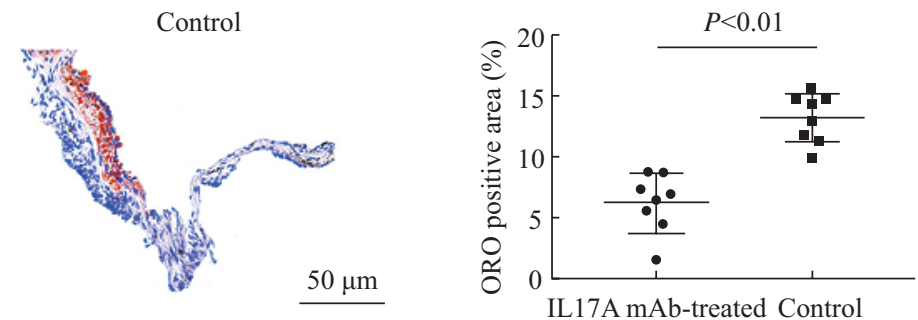
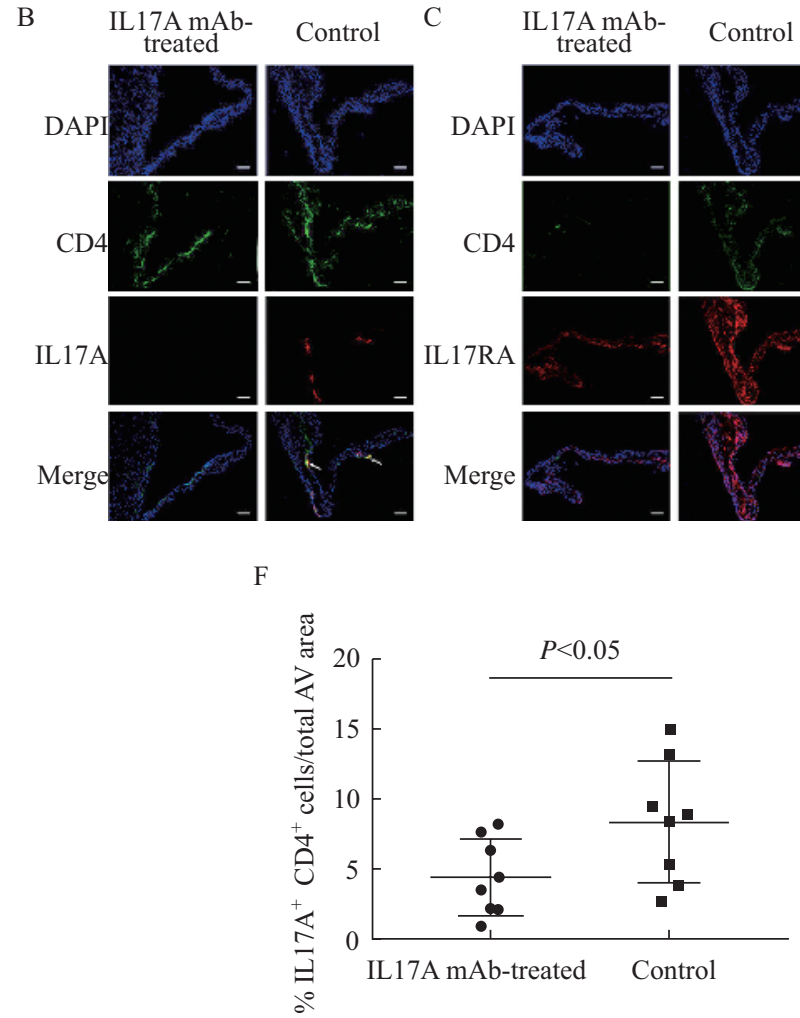

D

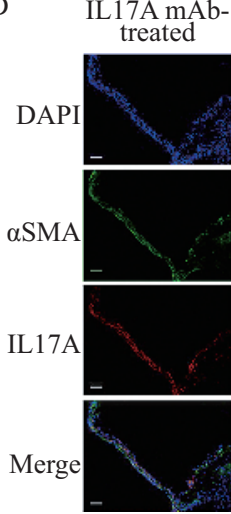

IL17A mAb-treated Control

G

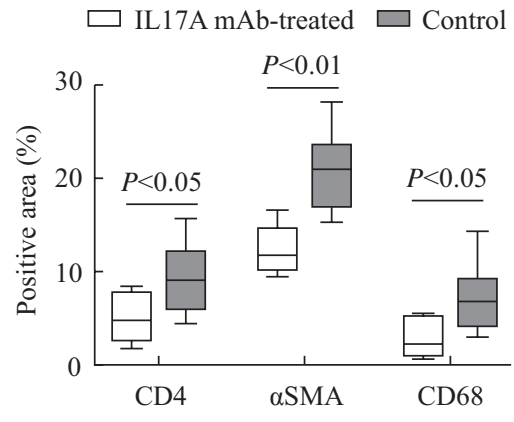

$\mathrm{H}$

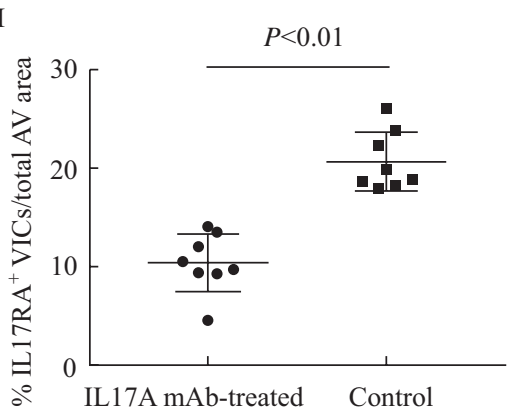

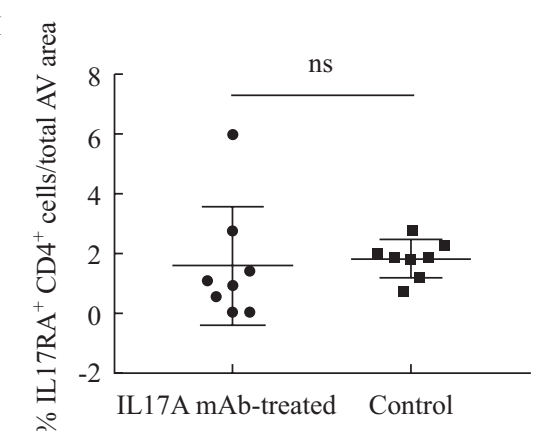

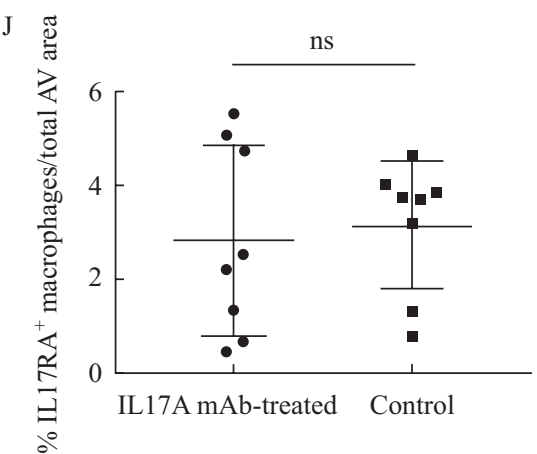

Fig. 2 Blocking IL17A alleviated inflammatory state of aortic valve of high-fat diet ApoE ${ }^{-/}$mice

Lipid deposition and cellular composition of aortic valve were compared. A: Oil Red O staining in aortic valves of IL17A $\mathrm{mAb}$-treated and control mice was presented $(n=8$ per group; $P<0.01)$. IL17A mAb-treatment reduced lipid deposition in HFD aortic valve as compared with controls. B-E double immunofluorescence staining images of IL17A/CD4 (B), IL17RA/CD4 (C), IL17RA/ $\alpha$ SMA (D), IL17RA/CD68 (E). F-J: quantification results ( $n=8$ per group). Treatment with IL17A mAb could significantly decrease the number of IL17 $\mathrm{A}^{+} \mathrm{CD} 4^{+} \mathrm{T}$ cells (F) (IL17A and CD4 double positive area, $P<0.05$ ), $\mathrm{CD} 4^{+} \mathrm{T}_{\text {cells }}\left(\mathrm{CD} 4^{+}\right.$ area, $P<0.05)$, macrophages (CD68 ${ }^{+}$area, $\left.P<0.05\right)$ and myofibroblastic VICs $\left(\alpha \mathrm{SMA}^{+}\right.$area, $\left.P<0.01\right)(\mathrm{G})$. Immunofluorescence colocalization analysis revealed a dropped number of IL17RA ${ }^{+}$VICs (H) (IL17RA and $\alpha$ SMA double positive area, $P<0.01$ ) in aortic valve of IL17A mAb-treated mice as compared with control mice. However, IL17RA ${ }^{+} \mathrm{CD}^{+}{ }^{+} \mathrm{T}$ cells (I) and IL17RA ${ }^{+}$ macrophages $(\mathrm{J})$ in IL17A mAb-treated groups showed no significant difference from control group. $20 \times$ magnification, scale bar $=40 \mu \mathrm{m} ; 40 \times$ magnification, scale bar $=50 \mu \mathrm{m}$

cell particle percentage of IL17RA ${ }^{+}$VICs and IL17RA ${ }^{+}$ macrophages in total aortic valve, we noticed HFD also

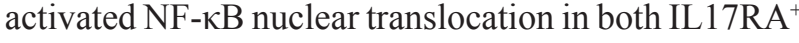
VICs (fig. 3A) and IL17RA ${ }^{+}$macrophages (fig. 3B). 


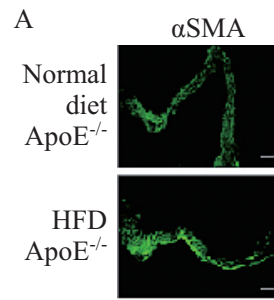

DAPI

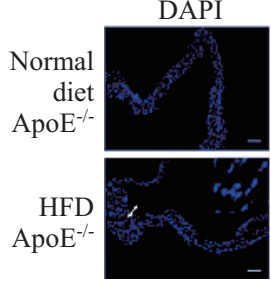

C
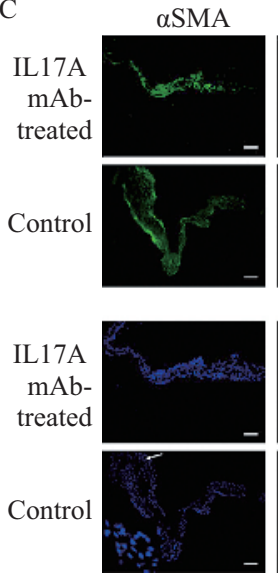

E

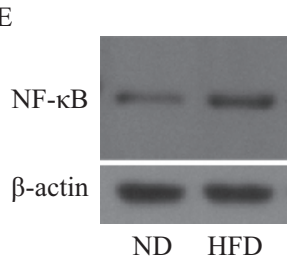

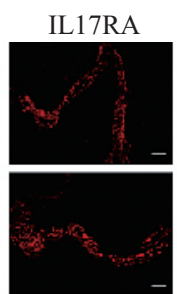

$\mathrm{NF}-\mathrm{kB}$
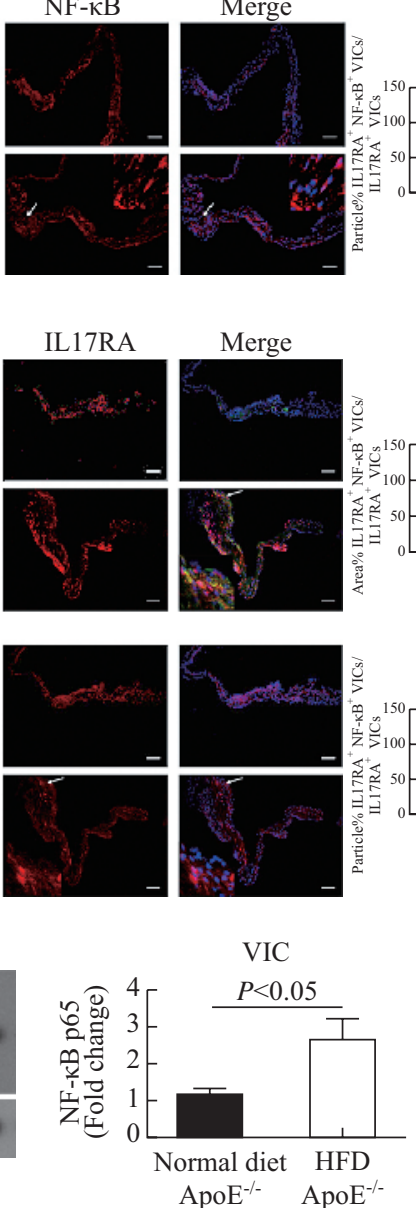
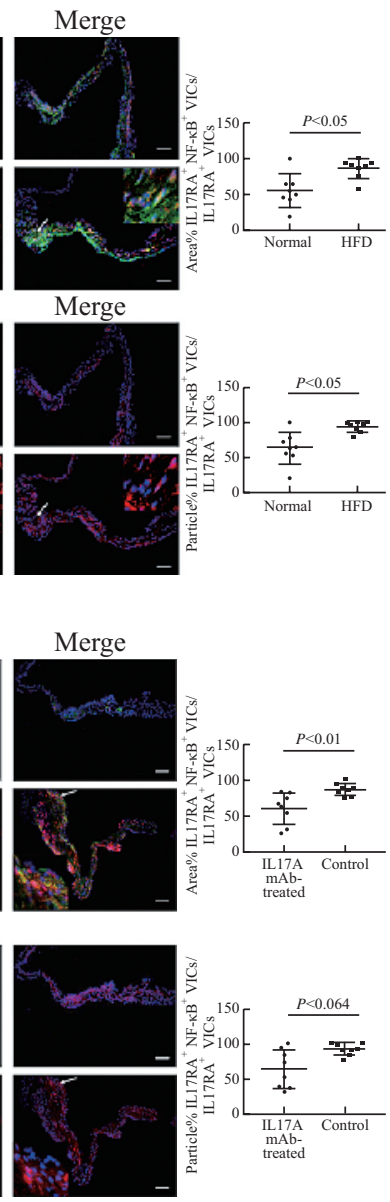

$\mathrm{D}$
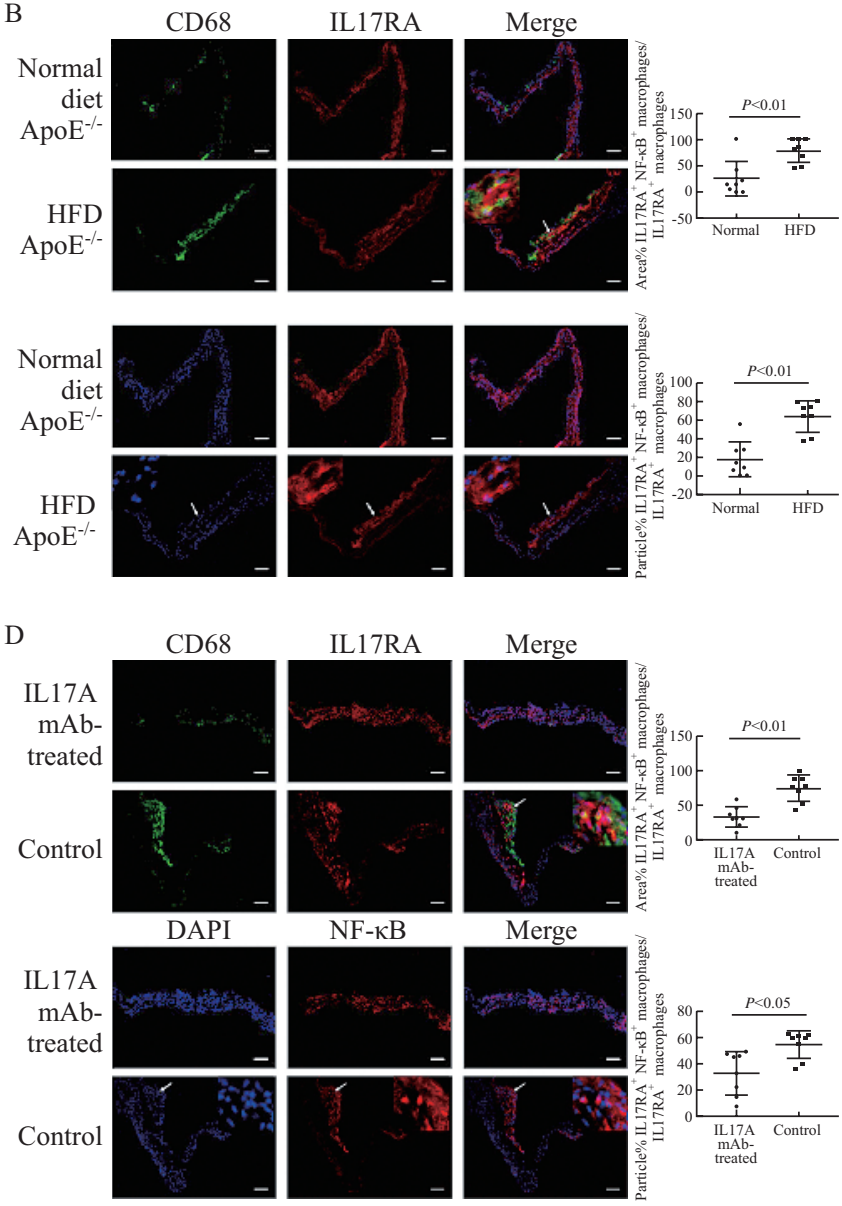

F
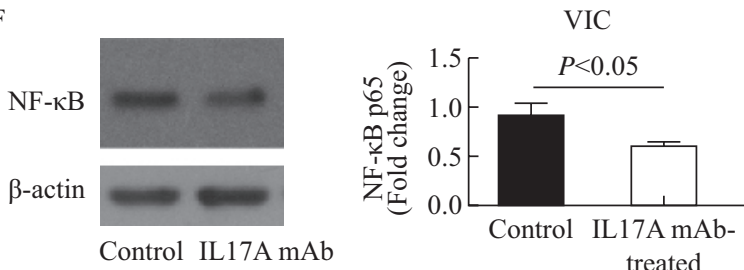

Fig. 3 IL17A/IL17RA were involved in aortic valve inflammation through NF- $\kappa B$ signaling pathway

Immunofluorescence analysis of NF- $\kappa B$ nuclear translocation in IL17RA ${ }^{+}$VICs and IL17RA ${ }^{+}$macrophages was performed. Normal diet (ND) ApoE ${ }^{-/-}$mice were compared with their high-fat diet (HFD) counterparts, whereas IL17A mAb-treated group was compared with irrelevant IgG-treated group. Translocation of NF- $\kappa B$ nuclear was assessed by NF- $\kappa B$ staining (arrows). IL17RA $^{+}$VICs and IL17RA ${ }^{+}$macrophages were respectively imaged by merged colors of $\alpha \mathrm{SMA}^{+}$IL17RA $^{+}$DAPI (A, C),

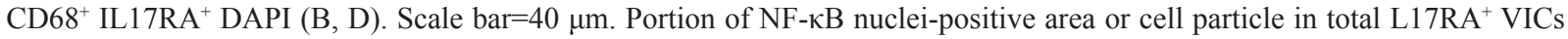
or IL17RA ${ }^{+}$macrophages was set as criteria to estimate NF- $\kappa B$ nuclear translocation. Separately, immunofluorescent staining images suggested obvious intranuclear translocation of NF- $\mathrm{BB}$ in IL17RA ${ }^{+}$VICs $(\mathrm{A})(P<0.05)$ and IL17RA ${ }^{+}$macrophages (B) $(P<0.01)$ in HFD group as compared with ND group. Meanwhile, IL17A mAb treatment illustrated blocking of NF- $\kappa \mathrm{B}$ intranuclear translocation in IL17RA ${ }^{+}$VICs (C) (area $\%, P<0.01$; particle $\left.\%, P=0.064\right)$ and IL17RA ${ }^{+}$macrophages (D) (area $\%$, $P<0.01$; particle \%, $P<0.05$ ). E: HFD induced the increase of NF-kB, as shown by the increase in NF-kB levels in nuclear extract Compared to ND. F: IL17A mAb treatment inhibited intranuclear translocation of NF- $\kappa B$, as shown by the decrease in NF- $\kappa B$ levels in nuclear extract compared to control group. Data were analyzed using the Student's $t$ test.

However, after IL17A mAb treatment, the NF- $\mathrm{kB}$ translocation was depressed in IL17RA+ VICs (fig. 3C) and IL17RA ${ }^{+}$macrophages (fig. 3D), although NF-kB ${ }^{+}$ cells counting results showed no significant difference in IL17RA ${ }^{+}$VICs $(P=0.064$, fig. 3C).

The results were further confirmed in VICs by Western blotting as shown in fig. 3E and 3F. As VICs are the main component of aortic valve and contribute to normal function, HFD ApoE $\mathrm{E}^{-/}$mice led to significant $\mathrm{NF}-\mathrm{\kappa B}$ intranuclear translocation in VICs (fig. 3E). After IL17A mAb treatment, the NF- $\mathrm{KB}$ translocation was depressed (fig. 3F).

\subsection{Validation of IL17A and IL17RA Expression Data by Real-time RT-PCR}

The expression levels of IL17A-IL17RA genes were detected using qRT-PCR. The result showed that 
the expression levels of IL17RA $(P<0.01)$ and IL17A $(P<0.01)$ were significantly increased in HFD ApoE ${ }^{-/-}$ mice samples (fig. 4A and 4B). As compared with the
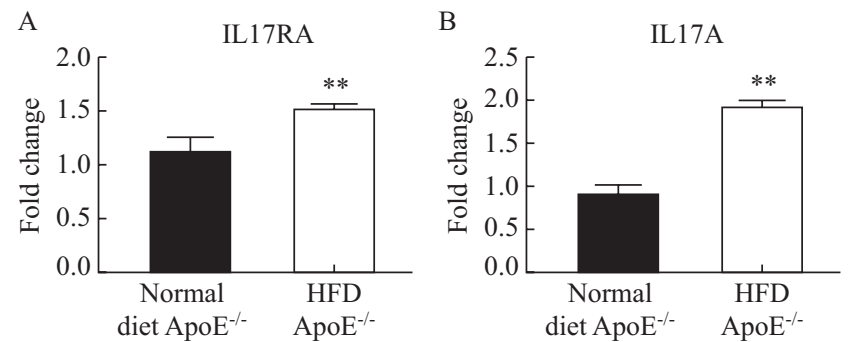

control group, IL17RA $(P<0.05)$ and IL17A $(P<0.01)$ were noticeably decreased in IL17A mAbtreated group (fig. 4C and 4D).
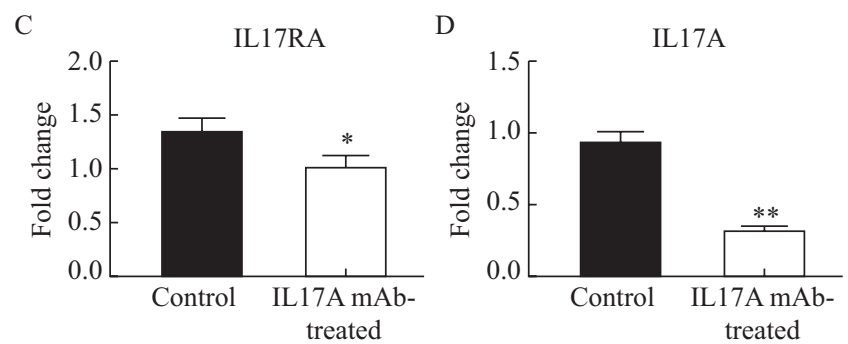

Fig. 4 qRT-PCR results of IL17A and IL17RA between normal diet and HFD ApoE-/ mice (A, B), and between IL17A mAb-treated and control mice $(\mathrm{C}, \mathrm{D})$. All data were normalized to GAPDH. $2^{-\triangle \Delta \mathrm{Ct}}$ methods were recruited to calculate the relative expression level. ${ }^{* *} P<0.01,{ }^{*} P<0.05$

\section{DISCUSSION}

Aortic valve disease is a significant culprit in cardiovascular disease and results from a complex interaction between hypercholesterolemia state and chronic inflammation. The development of aortic valve disease is characterized by three primary processes: lipid accumulation, inflammation, and ultimate calcification ${ }^{[23,24]}$. Inflammation is the core point to all stages of aortic valve pathology. Previous pathological studies suggested that stenotic aortic valve is rich in inflammatory cells and exhibits myofibroblastic differentiation in VICs - the most prevalent cells in aortic valve and responsible for maintaining normal valve structure and function. In inflammatory conditions, quiescent VICs will be activated into myofibroblasts ${ }^{[25,26]}$. $\alpha$ SMA, a cytoskeletal isoform of actin, is the phenotypic marker for VICs. The transition from VICs to myofibroblasts is also a critical change in valvular calcified pathology, markedly increasing the aSMA positive staining area. Overexpression of aSMA increases the VICs contractility and calcific nodule formation, whereas knockdown of aSMA with siRNAs reverses these changes ${ }^{[27]}$. Myofibroblastic transition is thought to contribute directly to the thickening and stiffening of valve and the subsequent calcification ${ }^{[28]}$. $\mathrm{CD}^{+} \mathrm{T}$ cells and macrophages are the principal infiltrated inflammatory cell types in various valvular heart diseases ${ }^{[29]}$. Inflammatory macrophages, as indicated by $\mathrm{CD} 68$, promote valvular cells calcification in a cathepsin S-dependent manner in aortic valve inflammatory disease ${ }^{[30]}$. Our current study employed HFD ApoE ${ }^{-/}$mice model as a wellestablished model for aortic valve inflammation, and displayed the increase of cholesterol and IL17A during aortic valve inflammation. At the same time, we observed significantly increased infiltration of inflammatory cells such as macrophages and $\mathrm{CD}^{+}$ $\mathrm{T}$ cells, and myofibroblastic transition of VICs.
Suppressing IL17A with IL17A mAb weakened levels of these inflammatory responses.

IL17A, which is primarily secreted by Th17 cells, participates in local tissue inflammation via inducing release of proinflammatory cytokines and neutrophil-mobilizing chemokines in various cell types $^{[10]}$. IL17RA is expressed in smooth muscle cells under basal conditions ${ }^{[31]}$. In atherosclerotic disease, IL17A/IL17RA are involved in systemic and vascular inflammation in response to HFD and are implicated in the progression of the atherosclerotic plaque $^{[12]}$. However, the role of IL17A in different stages of aortic valve inflammation remains unknown. In the current study, our data revealed that IL17A ${ }^{+}$ $\mathrm{CD}^{+} \mathrm{T}$ cells were increased in HFD aortic valve. Immunofluorescent staining also stated that IL17RA was raised significantly in VICs. Blockade of IL17A reversed the changes. Taken together, these findings indicated that IL17A and IL17RA were involved in VICs myofibroblastic transition, and $\mathrm{CD}^{+}{ }^{+} \mathrm{T}$ cells and macrophages infiltration in aortic valve inflammatory state.

Colocalization analysis is a vital section of coexpression study. Unlike the usual dye-overlap method easily misled by subjectivity, it identifies protein pairs and calculates colocalization area. The same results may also be acquired by using flow cytometry, but it will run out of almost all the treasured tissues. Additionally, we can also inquire a statistically testable, single-value assessment ICQ between the stained protein pairs. We used this co-expression method to assess IL17RA colocalized in the specific cells, not just IL17RA positive area in the whole aortic valve. At last, we obtained the results that IL17RA was significantly activated in myofibroblastic VICs in the HFD aortic valves.

In our study, IL17A/IL17RA contribute to inflammation by mean of cellular infiltration and activation, e.g., $\mathrm{T}$ cells and macrophages infiltration 
and VICs myofibroblastic transition. Furtherly, it can be speculated that IL17A activates VICs via IL17RA and subsequently triggers activation of NF- $\mathrm{KB}$, thereby inducing NF-kB-dependent gene transcription, as also reported in some publications for several other cell type $^{[32,33]}{ }^{33}$. On the other hand, as the number of VICs is various in different experiment groups, in order to avoid the bias, we used (NF-kB ${ }^{+}$IL17RA $^{+}$VICs)/ (IL17RA ${ }^{+}$VICs)\% value, instead of NF- $\mathrm{KB}$ positive area or cells in the whole aortic valve, to assess NF- $\mathrm{kB}$ translocation. At last we illustrated that the NF- $\mathrm{kB}$ was responsible for IL17A/IL17RA-related inflammation in VICs on HFD or IL17A mAb treatment mice.

In conclusion, we demonstrated for the first time that IL17A/IL17RA were involved in VICs myofibroblastic transition and inflammatory cells infiltration in the HFD ApoE ${ }^{-/}$mice, which is the downstream regulation mediated by NF- $\mathrm{kB}$. These conditions were reversible by inhibition of IL17A. Therefore, blocking IL17A could probably provide potential prophylactic and therapeutic potent for different stages of aortic valve diseases in the future.

\section{Open Access}

This article is licensed under a Creative Commons Attribution 4.0 International License https:/creativecommons.org/licenses/by/4.0/), which permits use, sharing, adaptation, distribution and reproduction in any medium or format, as long as you give appropriate credit to the original author(s) and the source, provide a link to the Creative Commons licence, and indicate if changes were made. The images or other third party material in this article are included in the article's Creative Commons licence, unless indicated otherwise in a credit line to the material. If material is not included in the article's Creative Commons licence and your intended use is not permitted by statutory regulation or exceeds the permitted use, you will need to obtain permission directly from the copyright holder. To view a copy of this licence, visit http://creativecommons.org/licenses/by/4.0/.

\section{Conflict of Interest Statement}

The authors declare no conflict of interest.

\section{REFERENCES}

1 Iung B, Vahanian A. Epidemiology of valvular heart disease in the adult. Nat Rev Cardiol, 2011,8(3):162172

2 Nkomo VT, Gardin JM, Skelton TN, et al. Burden of valvular heart diseases: a population-based study. Lancet, 2006, 368(9540):1005-1011

3 Smith CR, Leon MB, Mack MJ, et al. Transcatheter versus surgical aortic-valve replacement in high-risk patients. N Engl J Med, 2011,364(23):2187-2198

4 Melby SJ, Moon MR, Lindman BR, et al. Impact of pulmonary hypertension on outcomes after aortic valve replacement for aortic valve stenosis. J Thorac Cardiovasc Surg, 2011,141(6):1424-1430

5 Lindman BR, Patel JN. Multimorbidity in Older Adults with Aortic Stenosis. Clin Geriatr Med, 2016,32(2):305-
314

6 Novaro GM, Katz R, Aviles RJ, et al. Clinical factors, but not C-reactive protein, predict progression of calcific aortic-valve disease: the Cardiovascular Health Study. J Am Coll Cardiol, 2007,50(20):1992-1998

7 Cai Z, Li F, Gong W, et al. Endoplasmic reticulum stress participates in aortic valve calcification in hypercholesterolemic animals. Arterioscler Thromb Vasc Biol, 2013,33(10):2345-2354

8 Jung JJ, Razavian M, Challa AA, et al. Multimodality and molecular imaging of matrix metalloproteinase activation in calcific aortic valve disease. J Nucl Med, 2015,56(6):933-938

9 Sikura KE, Potor L, Szerafin T, et al. Hydrogen sulfide inhibits calcification of heart valves; implications for calcific aortic valve disease. $\mathrm{Br} \mathrm{J}$ Pharmacol, 2020,177(4):793-809

10 Kolls JK, Linden A. Interleukin-17 family members and inflammation. Immunity, 2004,21(4):467-476

11 Vugmeyster Y, Zhang YE, Zhong X, et al. Pharmacokinetics of anti-IL17A and anti-IL22 peptideantibody bispecific genetic fusions in mice. Int Immunopharmacol, 2014,18(2):225-227

12 Chen S, Shimada K, Zhang W, et al. IL-17A is proatherogenic in high-fat diet-induced and Chlamydia pneumoniae infection-accelerated atherosclerosis in mice. J Immunol, 2010,185(9):5619-5627

13 Smith E, Prasad KM, Butcher M, et al. Blockade of interleukin-17A results in reduced atherosclerosis in apolipoprotein E-deficient mice. Circulation, 2010, 121(15):1746-1755

14 Madhur MS, Funt SA, Li L, et al. Role of interleukin 17 in inflammation, atherosclerosis, and vascular function in apolipoprotein e-deficient mice. Arterioscler Thromb Vasc Biol, 2011,31(7):1565-1572

15 Eid RE, Rao DA, Zhou J, et al. Interleukin-17 and interferon-gamma are produced concomitantly by human coronary artery-infiltrating $\mathrm{T}$ cells and act synergistically on vascular smooth muscle cells. Circulation, 2009, 119(10):1424-1432

16 Choi B, Lee S, Kim SM, et al. Dipeptidyl Peptidase-4 Induces Aortic Valve Calcification by Inhibiting InsulinLike Growth Factor-1 Signaling in Valvular Interstitial Cells. Circulation, 2017,135(20):1935-1950

17 Zhang X, Liu F, Bai P, et al. Identification of key genes and pathways contributing to artery tertiary lymphoid organ development in advanced mouse atherosclerosis. Mol Med Rep, 2019,19(4):3071-3086

18 Li Q, Lau A, Morris TJ, i. A syntaxin 1, Galpha(o), and $\mathrm{N}$-type calcium channel complex at a presynaptic nerve terminal: analysis by quantitative immunocolocalization. J Neurosci, 2004,24(16):4070-4081

19 Steiner I, Krbal L, Rozkos T, et al. Calcific aortic valve stenosis: Immunohistochemical analysis of inflammatory infiltrate. Pathol Res Pract, 2012, 208(4): 231-234

20 Porras AM, Westlund JA, Evans AD, et al. Creation of disease-inspired biomaterial environments to mimic pathological events in early calcific aortic valve disease. Proc Natl Acad Sci USA, 2018,115(3):E363-E371

21 Erbel C, Chen L, Bea F, et al. Inhibition of IL-17A attenuates atherosclerotic lesion development in apoE- 
deficient mice. J Immunol, 2009,183(12):8167-8175

22 Zeng Q, Jin C, Ao L, et al. Cross-talk between the Tolllike receptor 4 and Notch1 pathways augments the inflammatory response in the interstitial cells of stenotic human aortic valves. Circulation, 2012,126(11 Suppl 1): S222-230

23 Yu PJ, Skolnick A, Ferrari G, et al. Correlation between plasma osteopontin levels and aortic valve calcification: potential insights into the pathogenesis of aortic valve calcification and stenosis. J Thorac Cardiovasc Surg, 2009,138(1):196-199

24 Izquierdo-Gomez MM, Hernandez-Betancor I, GarciaNiebla J, et al. Valve Calcification in Aortic Stenosis: Etiology and Diagnostic Imaging Techniques. Biomed Res Int, 2017,2017:5178631

25 Gould ST, Srigunapalan S, Simmons CA, et al. Hemodynamic and cellular response feedback in calcific aortic valve disease. Circ Res, 2013, 113(2):186-197

26 Rajamannan NM, Evans FJ, Aikawa E, et al. Calcific aortic valve disease: not simply a degenerative process: A review and agenda for research from the National Heart and Lung and Blood Institute Aortic Stenosis Working Group. Executive summary: Calcific aortic valve disease-2011 update. Circulation, 2011,124(16):17831791

27 Benton JA, Kern HB, Leinwand LA, et al. Statins block calcific nodule formation of valvular interstitial cells by inhibiting alpha-smooth muscle actin expression.
Arterioscler Thromb Vasc Biol, 2009,29(11):1950-1957

$28 \mathrm{Li} \mathrm{C}, \mathrm{Xu} \mathrm{S}$, Gotlieb AI. The progression of calcific aortic valve disease through injury, cell dysfunction, and disruptive biologic and physical force feedback loops. Cardiovasc Pathol, 2013,22(1):1-8

29 Wallby L, Steffensen T, Jonasson L, et al. Inflammatory Characteristics of Stenotic Aortic Valves: A Comparison between Rheumatic and Nonrheumatic Aortic Stenosis. Cardiol Res Pract, 2013,2013:895215

30 Aikawa E, Aikawa M, Libby P, et al. Arterial and aortic valve calcification abolished by elastolytic cathepsin $\mathrm{S}$ deficiency in chronic renal disease. Circulation, 2009, 119(13):1785-1794

31 Cheng G, Wei L, Xiurong W, et al. IL-17 stimulates migration of carotid artery vascular smooth muscle cells in an MMP-9 dependent manner via p38 MAPK and ERK1/2-dependent NF-kappaB and AP-1 activation. Cell Mol Neurobiol, 2009,29(8):1161-1168

32 Jovanovic DV, Di Battista JA, Martel-Pelletier J, et al. IL-17 stimulates the production and expression of proinflammatory cytokines, IL-beta and TNF-alpha, by human macrophages. J Immunol, 1998,160(7):35133521

33 Schwandner R, Yamaguchi K, Cao Z. Requirement of tumor necrosis factor receptor-associated factor (TRAF)6 in interleukin 17 signal transduction. J Exp Med, 2000,191(7):1233-1240

(Received June 8, 2019; accepted June 10, 2020) 